\title{
Myocardial infarction assessment by surface electrocardiography
}

\author{
Haren Patel, MD, ${ }^{\mathrm{a}, \mathrm{b}}$ Harish Doppalapudi, $M \mathrm{D}^{\mathrm{a}, \mathrm{b}}$ \\ and Fadi G. Hage, MD, MASNC \\ a Division of Cardiovascular Disease, Department of Medicine, University of Alabama at \\ Birmingham, Birmingham, AL \\ b Section of Cardiology, Birmingham Veterans Affairs Medical Center, Birmingham, AL
}

Received Sep 11, 2019; accepted Sep 12, 2019

doi: $10.1007 / \mathrm{s} 12350-019-01903-9$

\section{See related article, pp. 1364-1373}

Surface electrocardiography (ECG) is often used clinically for the detection of myocardial infarction (MI). Although prior guidelines used to classify MI as Q-wave and non-Q-wave MIs, current guidelines rely on ST segment shift on the ECG to differentiate ST-elevation MI from non-ST elevation acute coronary syndrome since that has direct impact on clinical management ${ }^{1}$. Further, we now realize that transmural MI may occur without $\mathrm{Q}$ waves on the ECG and nontransmural MIs may be accompanied by $Q$ waves ${ }^{2}$. Nevertheless, the presence of $Q$ waves on an ECG is helpful to suggest the presence of prior MI. In this regards, not all Q waves on ECG tracings are pathologic; pathologic Q waves are defined in the Fourth Universal Definition of Myocardial Infarction as the presence of $Q$ wave in leads V2-V3 $>0.02 \mathrm{~s}$ or QS complex in leads V2-V3; Q wave $\geq 0.03 \mathrm{~s}$ and $\geq 1 \mathrm{~mm}$ deep or QS complex in leads I, II, aVL, aVF, or V4-V6 in any 2 leads of a contiguous lead grouping (I, aVL; V3-V6; II, III, aVF); or an R wave $>0.04 \mathrm{~s}$ in V1-V2 and R/S $>1$ with a concordant positive $\mathrm{T}$ wave in absence of conduction defect ${ }^{3}$. Even pathologic $Q$ waves may be present in the absence of MI. Notoriously, lead misplacement can result in the misdiagnosis of MI by ECG. Any condition that induces a shift in the position of the

Reprint requests: Haren Patel, MD, Division of Cardiovascular Disease, Department of Medicine, University of Alabama at Birmingham, LHRB 326, 701 19th Street South, Birmingham, AL 35294, USA; harenpatel@uabmc.edu

J Nucl Cardiol 2021;28:1374-7.

$1071-3581 / \$ 34.00$

Copyright (C) 2019 American Society of Nuclear Cardiology. heart in relation to the surface ECG leads can have similar consequences. Other conditions that have been associated with Q waves in the absence of MI include Wolff-Parkinson-White syndrome, amyloid heart disease, hypertrophic cardiomyopathy, non-ischemic dilated cardiomyopathy, chronic obstructive pulmonary disease, left bundle branch block, and paced rhythm ${ }^{2}$.

The gold standard for diagnosis of MI is the presence of myocardial cell death due to prolonged ischemia on autopsy ${ }^{3}$. Current imaging studies allow for the diagnosis of $\mathrm{MI}$ in a relatively non-invasive manner. The presence of myocardial scar by imaging is known to correlate with poor outcomes ${ }^{4,5}$. The identification of viable (but non-functional) myocardium on imaging suggests that coronary revascularization to that region of the heart may result in the recovery of myocardial function ${ }^{6}$. In contrast, the restoration of blood flow to scarred myocardium is unlikely to be associated with clinical benefit and may result in harm due to the elevated risk associated with coronary revascularization in these patients. Positron emission tomography (PET) imaging using flow (ex: ${ }^{13} \mathrm{~N}$-ammonia) and metabolic $\left({ }^{18} \mathrm{~F}\right.$-fluorodeoxyglucose-FDG) tracers is a very accurate method for the identification of scar and myocardial viability ${ }^{7}$.

In this issue of the Journal, Markendorf and colleagues evaluated the ability of ECG to predict the presence of myocardial scar using cardiac PET as gold standard $^{8}$. They studied 149 patients known to have ischemic cardiomyopathy defined by evidence of coronary artery stenosis on prior angiography or a history of MI who underwent ammonia and FDG PET at a single institution. They evaluated the correlation of $\mathrm{Q}$ and/or QS waves on ECG with scarred myocardium by PET defined as a region of matching defect ( $<50 \%$ uptake) on FDG and ammonia PET images. The presence of Q waves was associated with a higher number of scarred 
segments on PET (2.0 vs 1.7). On a per patient analysis, the sensitivity, specificity, positive predictive value (PPV), and negative predictive value (NPV) of Q waves for detecting scar were $70 \%, 40 \%, 37 \%$, and $73 \%$ respectively. When using only QS complexes, the corresponding vales were $46 \%, 59 \%, 36 \%$, and $68 \%$ respectively. On a per vessel analysis these were 56\%, $73 \%, 25 \%$, and $91 \%$, respectively, for $\mathrm{Q}$ waves and $33 \%, 87 \%, 30 \%$, and $89 \%$, respectively, for only QS complexes. The authors conclude that although the negative predictive value may be at least modest, the presence of Q or QS complexes on ECG should not be used to determine the presence of myocardial scar or to guide in whom coronary revascularization should or shouldn't be sought ${ }^{8}$.

The utility of an ECG to detect MI has been extensively studied with the consensus that it is relatively unreliable and insensitive in this regard as even those with prior MI could have normal resting ECGs ${ }^{9-11}$ Jaarsma et al evaluated different scoring systems for the detection of prior MI and found at best modest diagnostic accuracy using late gadolinium enhancement cardiovascular magnetic resonance (CMR) imaging as gold standard. They cautioned against the exclusion of previous MI based on ECG criteria alone ${ }^{12}$. In a different study, Kakhi and colleagues reported a sensitivity of only $61 \%$ for $\mathrm{Q}$ waves on ECG to detect MI with single emission computed tomography (SPECT) myocardial perfusion imaging (MPI) as the gold standard ${ }^{13}$.

The Minnesota Code is a guide to systematically critique an ECG in order to identify pathology. It has gone through multiple revisions and is generally used in studies to standardize the definition of ECG findings. In an interesting study, Asch et al evaluated the accuracy of ECG Q or QS waves to detect prior MI using CMR as gold standard. The Minnesota Code definition of $\mathrm{Q}$ or QS waves on ECG had a sensitivity of only $48 \%$ for the detection of scar comprising more than $1 \%$ of the total ventricular volume by CMR. The specificity was $84 \%$. The positive and negative predictive values were $73 \%$ and 64\%, respectively. Although the sensitivity increased with increasing size of scar by CMR, the increase was modest at best reaching $64 \%$ for scars larger than $30 \%$ of left ventricular volume (this decreased the specificity to $73 \%$ ). Whether the scar is transmural or not, the accuracy of ECG was not altered. When analyzing the results by location of the scar, the sensitivity was lowest for lateral scars with only $14 \%$ of those detected by $\mathrm{ECG}^{14}$. Nadour et al reported similar results (also using CMR as reference) with a sensitivity of $66 \%$ and specificity of $85 \%$ with a significant number of false positives of $\mathrm{Q}$ waves in the absence of CMR scar findings ${ }^{15}$.
Sandler et al used thallium SPECT MPI as a reference standard and the Minnesota Code criteria for $\mathrm{Q}$ and QS complexes on ECG. They reported a sensitivity of $58 \%$ and specificity of $75 \%$. The specificity was improved by using more stringent criteria on the Minnesota Code at the expense of lower sensitivity (sensitivity of $30 \%$ for the most stringent criteria) ${ }^{16}$. Taneja et al evaluated the accuracy of Q waves on ECG to predict myocardial viability (using dobutamine stress echocardiography as reference) in patients who had recent MI. They reported a sensitivity of $64 \%$ and specificity of $70 \%$. Thus, the presence of $\mathrm{Q}$ waves did not predict non-viable myocardium ${ }^{17}$. Al-Mohammad et al asked a similar question to Markendorf and colleagues, but did so in a smaller patient population, evaluating the ability of $\mathrm{Q}$ waves to detect viability based on PET imaging. They reported a sensitivity of $41 \%$ and a specificity of $79 \%$. Although they reported a lower sensitivity than specificity (unlike in the current report by Markendorf ${ }^{8}$ ), both were only modest at best ${ }^{18}$.

It is important to recognize that these studies were largely performed in patients suspected of having an MI and undergoing advanced imaging. As detailed above in the study by Markendorf et $\mathrm{al}^{8}$, the included patients had a history of ischemic cardiomyopathy and were undergoing imaging with PET for assessment of myocardial viability. The majority of these patients $(65 \%)$ had a clinical history of MI (if the clinical history of MI is the gold standard, even the most sensitive imaging test has low accuracy for its detection). How these data on the accuracy of the ECG translate to patients not suspected of having an MI is not clear. This is an important question given the prevalence of 'silent MI' using ECG criteria documented in several cohorts. Multiple studies have also confirmed that silent MI by ECG confers a poor outcome that may be comparable to clinical $\mathrm{MI}^{19-21}$. In this regards, Soliman et al highlighted the importance of having a systematic approach to interpreting the ECG for presence of an MI given the wide range of incidence and prevalence reported in the literature when using the ECG alone to determine the presence of prior $\mathrm{MI}^{22}$.

We congratulate Markendorf et $\mathrm{al}^{8}$ on the performance of this study and adding to the body of literature on the accuracy of ECG for the detection of MI. The ECG can be obtained quickly, is readily available, and relatively cheap, and its performance is not associated with adverse events, but it has significant limitations. As detailed above (summarized in Table 1) and highlighted by Markendorf et $\mathrm{al}^{8}$, the diagnostic accuracy of the ECG for detection of MI is suboptimal. In our opinion, the identification of MI by ECG should alert the clinician for the possibility of prior MI if not otherwise suspected. This should be followed by imaging tests to 
Table 1. Summary of studies assessing the ECG vs various imaging modalities

\begin{tabular}{lllccc}
\hline Authors & \multicolumn{1}{c}{ EKG finding } & $\begin{array}{c}\text { Imaging } \\
\text { modality }\end{array}$ & $\begin{array}{c}\text { Number } \\
\text { of patients }\end{array}$ & $\begin{array}{c}\text { Sensitivity } \\
\text { (\%) }\end{array}$ & $\begin{array}{c}\text { Specificity } \\
\text { (\%) }\end{array}$ \\
\hline Markendorf & Q waves & PET & 149 & 70 & 40 \\
Jarrasma & Universal definition of previous MI & MRI & 114 & 33 & 97 \\
Jaarsma & Minnesota ECG code & MRI & 114 & 79 & 77 \\
Jaarsma & Selvester QRS score & MRI & 114 & 90 & 71 \\
Jaarsma & Cardiologist assessment & MRI & 114 & 67 & 74 \\
Kakhi & Q waves & SPECT & 476 & 61 & 94 \\
Kakhi & Fragmented QRS complex & SPECT & 476 & 78 & 65 \\
Asch & Q waves & MRI & 139 & 48.4 & 83.5 \\
Nadour & Q waves & MRI & 235 & 66 & 85 \\
Sandler & Minnesota ECG code & SPECT & 214 & 58 & 75 \\
Al-Mohammad & Q waves & PET & 58 & 41 & 79 \\
Taneja & Q waves & Echo & 176 & 64 & 70 \\
\hline
\end{tabular}

confirm or refute that assumption. As stated earlier, ST segment shift on the ECG has direct impact on current management of acute coronary syndrome. However, in the modern era, the ECG should not be used in isolation for the diagnosis of prior MI and the presence or absence of ECG criteria for MI should not alone dictate clinical management.

\section{Disclosure}

Dr. Hage reports investigator initiated grant support from Astellas Pharma. The other authors have no conflicts of interest to disclose. The work was not supported by industry.

\section{References}

1. Amsterdam EA, Wenger NK, Brindis RG, et al. 2014 AHA/ACC guideline for the management of non-ST-elevation acute coronary syndromes: Executive summary: A report of the American College of Cardiology/American Heart Association Task Force on Practice Guidelines. Circulation 2014;130:2354-94.

2. Zipes DP, Libby P, Bonow R, et al. Braunwald's heart disease: A textbook of cardiovascular medicine. Philadelphia, PA: Elsevier; 2019.

3. Thygesen K, Alpert JS, Jaffe AS, et al. Fourth universal definition of myocardial infarction (2018). J Am Coll Cardiol 2018;72:223164.

4. Hage FG, Ghimire G, Lester D, et al. The prognostic value of regadenoson myocardial perfusion imaging. J Nucl Cardiol 2015;22:1214-21.

5. Hage FG, Venkataraman R, Aljaroudi W, et al. The impact of viability assessment using myocardial perfusion imaging on patient management and outcome. J Nucl Cardiol 2010;17:37889.

6. Dilsizian V, Bacharach SL, Beanlands RS, et al. ASNC imaging guidelines/SNMMI procedure standard for positron emission tomography (PET) nuclear cardiology procedures. J Nucl Cardiol 2016;23:1187-226.

7. Arrighi JA, Dilsizian V. Multimodality imaging for assessment of myocardial viability: Nuclear, echocardiography, MR, and CT. Curr Cardiol Rep 2012;14:234-43.

8. Markendorf S, Benz DC, Messerli M, et al. Value of 12-lead electrocardiogram to predict myocardial scar on FDG PET in heart failure patients. J Nucl Cardiol 2019

9. Uusitupa M, Pyörälä K, Raunio H, et al. Sensitivity and specificity of Minnesota Code Q-QS abnormalities in the diagnosis of myocardial infarction verified at autopsy. Am Heart $\mathrm{J}$ 1983;106:753-7.

10. Martinez-Rios MA, Da Costa BC, Cecena-Seldner FA, et al. Normal electrocardiogram in the presence of severe coronary artery disease. Am J Cardiol 1970;25:320-4.

11. Bär FW, Brugada $P$, Dassen WR, et al. Prognostic value of $Q$ waves, R/S ratio, loss of R wave voltage, ST-T segment abnormalities, electrical axis, low voltage and notching: correlation of electrocardiogram and left ventriculogram. J Am Coll Cardiol 1984;4:17-27.

12. Jaarsma C, Bekkers SC, Haidari Z, et al. Comparison of different electrocardiographic scoring systems for detection of any previous myocardial infarction as assessed with cardiovascular magnetic resonance imaging. Am J Cardiol 2013;112:1069-74.

13. Kakhi VR, Ayati N, Zakavi SR, et al. Comparison between fragmented QRS and Q waves in myocardial scar detection using myocardial perfusion single photon emission computed tomography. Kardiol Pol 2015;73:437-44.

14. Asch FM, Shah S, Rattin C, et al. Lack of sensitivity of the electrocardiogram for detection of old myocardial infarction: A cardiac magnetic resonance imaging study. Am Heart J 2006;152:742-8.

15. Nadour W, Doyle M, Williams RB, et al. Does the presence of Q waves on the EKG accurately predict prior myocardial infarction when compared to cardiac magnetic resonance using late gadolinium enhancement? A cross-population study of noninfarct vs infarct patients. Heart Rhythm 2014;11:2018-26.

16. Sandler LL, Pinnow EE, Lindsay J. The accuracy of electrocardiographic $Q$ waves for the detection of prior myocardial infarction as assessed by a novel standard of reference. Clin Cardiol 2004;27:97-100. 
17. Taneja AK, Hayat S, Swinburn J, et al. Usefulness of Q waves on ECG for the prediction of contractile reserve after acute myocardial infarction. Int J Cardiol 2010;145:265-6.

18. Al-Mohammad A, Norton MY, Mahy IR, et al. Can the surface electrocardiogram be used to predict myocardial viability? Heart 1999;82:663-7.

19. Gibson CM, Nafee T, Kerneis M. Silent myocardial infarction: Listen to the evidence. J Am Coll Cardiol 2018;71:9-11.

20. Zhang ZM, Rautaharju PM, Prineas RJ, et al. Race and sex differences in the incidence and prognostic significance of silent myocardial infarction in the atherosclerosis risk in communities (ARIC) study. Circulation 2016;133:2141-8.
21. Farag AA, AlJaroudi W, Neill J, et al. Prognostic value of silent myocardial infarction in patients with chronic kidney disease being evaluated for kidney transplantation. Int $\mathrm{J}$ Cardiol 2017;249:377-82.

22. Soliman EZ. Electrocardiographic definition of silent myocardial infarction in population studies: A call to standardize the standards. J Electrocardiol 2019;55:128-32.

Publisher's Note Springer Nature remains neutral with regard to jurisdictional claims in published maps and institutional affiliations. 